\title{
BMJ Open Effect of different communication strategies about stopping cancer screening on screening intention and cancer anxiety: a randomised online trial of older adults in Australia
}

\author{
Jenna Smith (D) , ${ }^{1}$ Rachael H Dodd (D) , ${ }^{1}$ Jolyn Hersch (1) , ${ }^{1}$ Erin Cvejic (D) , ${ }^{1}$ \\ Kirsten McCaffery, ${ }^{1}$ Jesse Jansen ${ }^{1,2}$
}

To cite: Smith J, Dodd RH, Hersch J, et al. Effect of different communication strategies about stopping cancer screening on screening intention and cancer anxiety: a randomised online trial of older adults in Australia. BMJ Open 2020;10:e034061. doi:10.1136/ bmjopen-2019-034061

- Prepublication history and additional material for this paper are available online. To view these files, please visit the journal online (http://dx.doi org/10.1136/bmjopen-2019034061).

Received 04 September 2019 Revised 09 February 2020 Accepted 19 May 2020

Check for updates

(c) Author(s) (or their employer(s)) 2020. Re-use permitted under CC BY-NC. No commercial re-use. See rights and permissions. Published by BMJ.

${ }^{1}$ School of Public Health, The University of Sydney, Sydney, New South Wales, Australia

${ }^{2}$ School for Public Health and Primary Care, Maastricht University, Maastricht, Netherlands

Correspondence to

Dr Jesse Jansen;

jesse.jansen@sydney.edu.au

\section{ABSTRACT}

Objective To assess different strategies for communicating to older adults about stopping cancer screening.

Design 4 (recommendation statement about stopping screening $) \times(2$; time) online survey-based randomised controlled trial.

\section{Setting Australia.}

Participants 271 English-speaking participants, aged 65-90, screened for breast/prostate cancer at least once in past decade.

Interventions Time 1: participants read a scenario in which their general practitioner (GP) informed them about the potential benefits and harms of cancer screening, followed by double-blinded randomisation to one of four recommendation statements to stop screening: control ('this screening test would harm you more than benefit you'), health status ('your other health issues should take priority'), life expectancy framed positively ('this test would not help you live longer') and negatively ('you may not live long enough to benefit'). Time 2: in a follow-up scenario, the GP explained why guidelines changed over time (anchoring bias intervention).

Measures Primary outcomes: screening intention and cancer anxiety (10-point scale, higher=greater intention/ anxiety), measured at both time points. Secondary outcomes: trust (in their GP, the information provided, the Australian healthcare system), decisional conflict and knowledge of the information presented.

Results 271 participants' responses analysed. No main effects were found. However, screening intention was lower for the negatively framed life expectancy versus health status statement (6.0 vs 7.1, mean difference $(M D)=1.1, p=0.049,95 \% \mathrm{Cl} 0.0$ to 2.2 ) in post hoc analyses. Cancer anxiety was lower for the negatively versus positively framed life expectancy statement (4.8 vs $5.8, \mathrm{MD}=1.0, \mathrm{p}=0.025,95 \% \mathrm{Cl} 0.1$ to 1.9 ). The anchoring bias intervention reduced screening intention $(\mathrm{MD}=0.8$, $\mathrm{p}=0.044,95 \% \mathrm{Cl} 0.6$ to 1.0$)$ and cancer anxiety $(\mathrm{MD}=0.3$, $\mathrm{p}=0.002,95 \% \mathrm{Cl} 0.1$ to 0.4 ) across all conditions. Conclusion Older adults may reduce their screening intention without reporting increased cancer anxiety when clinicians use a more confronting strategy communicating
Strengths and limitations of this study

- This randomised controlled trial used an experimental online design to test different strategies for communicating the counterintuitive idea of stopping cancer screening to older adults.

- Previous work has focused on what older adults prefer when it comes to discussing stopping screening; our study tests how incorporating health status and life expectancy into the conversation affects the decision process.

- We used hypothetical scenarios and measured screening intention rather than actual behaviour; individuals in a real-life setting may behave differently.

- Our study was possibly underpowered to detect significant differences.

they may not live long enough to benefit and add an explicit explanation why the recommendation has changed.

Trial registration number Australian New Zealand Clinical Trials Registry (ACTRN12618001306202; Results).

\section{INTRODUCTION}

Older adults, particularly those with limited life expectancy or poor health, are less likely to benefit from routine cancer screening because they may not live long enough for a screen-detected cancer to become problematic. ${ }^{1-3}$ For example, the benefits of regular prostate-specific antigen (PSA) testing are uncertain for men in general, but especially for those aged over 70 years ${ }^{4-6}$ and women aged over 74 years who routinely screen for breast cancer are also unlikely to benefit. ${ }^{7-9}$

Continued routine cancer screening in older adults can also lead to harm, including 
pain and anxiety related to mammography and PSA tests, the need for additional tests due to false-positive results, and overdiagnosis (having a cancer diagnosed and treated despite it not being destined to present clinically in the person's limited remaining lifetime).$^{5910}$ Overdiagnosis is an important harm as the side effects and complications of unneeded cancer treatment can lead to reduced quality of life in older people. ${ }^{11} 12$

Many international guidelines now recommend older people stop routine screening, especially those with limited life expectancy or poor health. Although not all American guidelines agree, the American Cancer Society, for example, suggests that women should continue screening as long as they are expected to live 10 or more years and are in good health. ${ }^{13}$ In Australia and Canada, women aged over 74 are no longer invited to screening (although they can still be screened on request). ${ }^{14} 15$ For PSA testing, Australian clinical practice guidelines recommend that men aged 50-69 years who want to be tested only undergo regular testing after being informed of the benefits and harms, ${ }^{16}$ and some guidelines in the USA and UK recommend against PSA testing altogether. ${ }^{4}$ Even so, many older adults aged 65 years or older with limited life expectancies (even less than 5 years) continue to screen for breast and prostate cancer. ${ }^{18} 19$

One reason for this is the decades of persuasive and unbalanced public health messaging about cancer screening that has made the idea of stopping screening seem counterintuitive and challenging to communicate. ${ }^{20}{ }^{21}$ Some older adults' attitudes toward screening are now a more important predictor of their screening decision than a clinician's recommendation. ${ }^{22}$ Additionally, health professionals hesitate to inform patients about confusing potential harms such as overdiagnosis ${ }^{23}$ and the majority (around $75 \%$ ) of older American adults recall no conversation with a doctor that screening for breast or prostate cancer may no longer be necessary. ${ }^{19}$ These conversations inherently involve sensitive and uncertain topics that clinicians feel uncomfortable to raise with their older patients. ${ }^{24} 25$

A qualitative study interviewed 40 men and women aged 65 years and older to identify their preferences for communicating about discontinuing screening. ${ }^{26}$ Participants preferred recommendations incorporating overall health status as the main reason for stopping screening and were divided about mentioning life expectancy. Specifically, the negatively framed statement 'you may not live long enough to benefit from this test' was seen as too harsh, with positive framing 'this test is not going to help you live longer' being preferred. These results were confirmed in a best-worst scaling survey from the same research group, with the health status statement ranked first and the positively and negatively framed life expectancy statements ranking 8 out of 13 and 12 out of 13, respectively. ${ }^{27}$

Although these previous studies highlight older adults' preferences for discussing stopping cancer screening, they did not examine how different communication strategies influence their decision-making and related outcomes. The primary aim of the current study was to experimentally test the effect of different strategies for communicating about stopping cancer screening to older adults on screening intention and cancer anxiety.

\section{METHODS}

\section{Trial design}

The online study used a $4 \times(2)$ mixed design with participants randomly assigned (stratified by gender) to one of four scenarios with a balanced allocation ratio of 1:1:1:1. The within-subjects element examined whether primary outcomes changed after the anchoring bias intervention, as described in more detail below. Pilot testing was conducted in the target population to ensure the study was realistic and suitable.

\section{Patient and public involvement}

No patient involved. However, acceptability of communication strategies was examined in previous qualitative work. $^{26}$

\section{Participants}

Participants were 65 years or older and living in Australia, recruited through Dynata, a digital data collection company experienced in panel survey sampling with an extensive database of participants. Dynata uses their existing panel of individuals who have signed up to receive surveys and targets participants based on certain parameters, such as gender, age, location and interests. Participation is incentivised using points, which participants can redeem for rewards such as cash, gift cards, charity donations, airline miles or virtual currencies. Eligible participants were proficient in English, had engaged in PSA testing (men) or mammography screening (women) at least once in the last decade (to ensure participants had a screening history) and had no dementia or breast/ prostate cancer diagnosis.

\section{Interventions}

Scenario describing a conversation with general practitioner (GP) about screening

Participants were presented with a scenario in which they were having a hypothetical conversation with their GP about cancer screening (PSA testing for men and mammography screening for women). The GP presented information about the potential harms of cancer screening, particularly for older people. Scenarios were based on a recent qualitative study, ${ }^{26}$ a decision aid for breast cancer screening, ${ }^{28}$ and advice from clinicians with expertise in general practice, cancer screening and decision-making (online supplementary material).

\section{Recommendation statement condition}

Participants were then randomly allocated to receive one of four statements recommending stopping screening (figure 1), based on Schoenborn et al $l$ s qualitative work. ${ }^{26}$ 


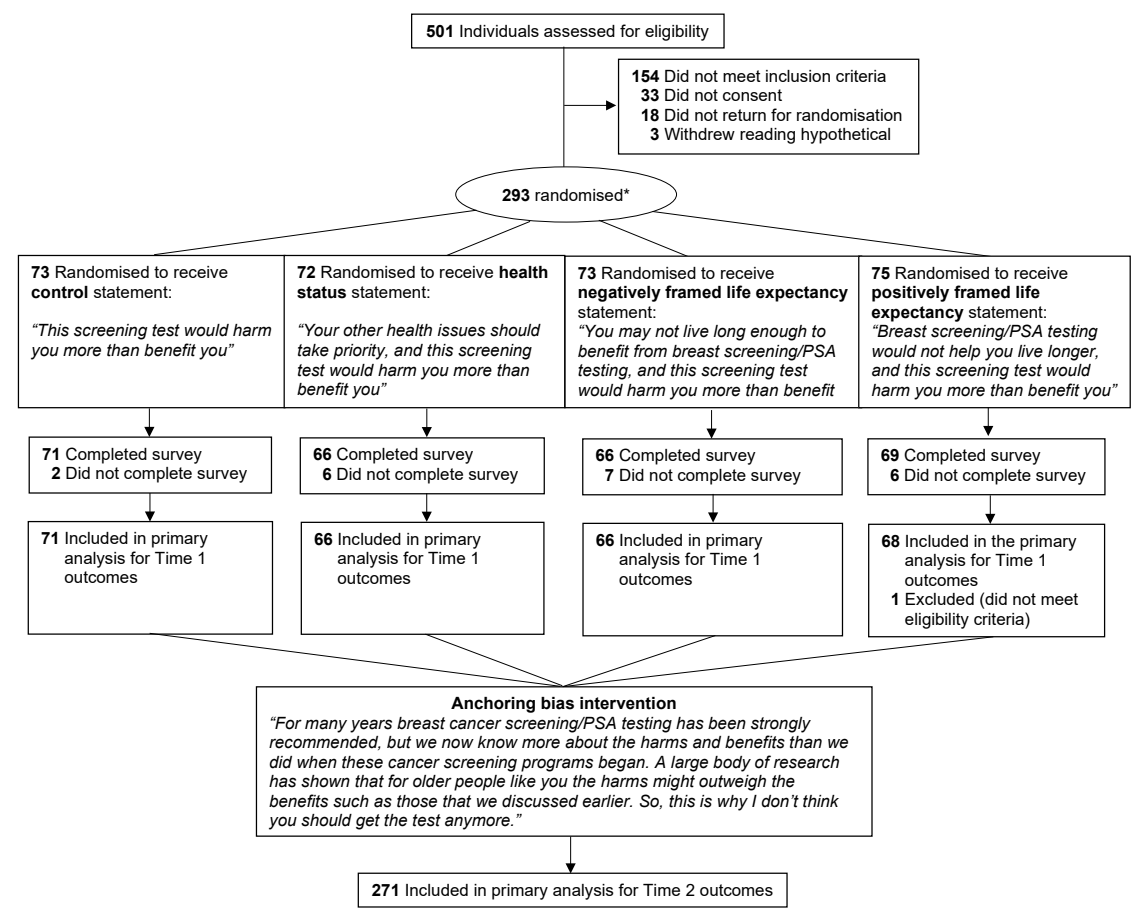

Figure 1 Participant flow and study design. *Randomisation stratified by gender whereby women read information about breast cancer and men read information about prostate cancer. PSA, prostate-specific antigen.

\section{Anchoring bias intervention}

All participants read a second scenario after completing the measures at time 1. This addressed the anchoring cognitive bias, which is a pre-existing belief (in this case, overwhelming positive views about cancer screening) that hinders the uptake of new, contradictory information (that stopping screening might be better in older age) ${ }^{29}$ In this scenario, the GP acknowledged the counterintuitive nature of the recommendation to stop screening and described why the recommendations have changed, in particular for older people (figure 1).

\section{Procedure}

The entire survey was created and delivered using Qualtrics software. Piloting was done to test the suitability and acceptability of the scenarios and questionnaire and no responses of concern were found. Participants were randomised automatically using the randomiser function included in Qualtrics, which uses the Mersenne Twister pseudorandom number generator. Participants and researchers were blinded to the allocation sequence until data collection was complete. After participants gave consent, they read the scenario describing a conversation with their GP, were randomised to one of the statements and then completed the questionnaire (time 1). All participants were then presented with the anchoring bias intervention and answered additional measures at time 2, as well as demographic questions.

\section{Outcomes}

Time 1 outcome measures

\section{Primary outcomes}

1. Screening intention: intention to have cancer screening was measured using a single item adapted from previous research: 'Based on this information provided to you by your GP, please indicate on the scale of 1-10 which best describes your intention to have the screening test'. ( 1 = 'Definitely will not' to $10=$ 'Definitely will'). ${ }^{30}$

2. Cancer anxiety: scores on two items were averaged to assess participants' level of anxiety about developing breast/prostate cancer: 'If this was really you, how worried would you be about the possibility of having breast/prostate cancer on a scale from 1 to 10 ?' and 'If you did not get this test, would you be so worried about the possibility of having breast/prostate cancer that you would worry much of the time'? $(1=$ 'Not at all' to $10=$ 'Very much so'). ${ }^{31}$

\section{Secondary outcomes}

1. Trust: three items were used to measure trust in the information provided by the GP, in their own GP and in the Australian healthcare system on a 5-point Likert scale ('Very little trust' to 'A great deal of trust'), adapted from a previous study. ${ }^{32}$

2. Decisional conflict: the low literacy version of the Decisional Conflict Scale: 10 items with 3 response options ('Yes', 'Unsure' or 'No') addressing four key aspects of conflict relating to patient decision-making (uncertainty, informed, values clarity and support), which are commonly interpreted independently. ${ }^{33-35}$ Higher scores indicate greater decisional conflict. 
3. Knowledge (about first scenario): single categorical item assessing understanding of the information: 'All breast/prostate cancers will eventually cause illness and death if they are not found and treated' ('True', 'False' and 'Don't know'). ${ }^{28}$

Time 2 outcome measures

Primary outcomes

1. Screening intention (repeated measure): 'Now you have been given this extra information, please indicate on the scale of 1-10 which best describes your intention to have the screening test'.

2. Cancer anxiety (repeated measure): same as time 1 .

\section{Secondary outcomes}

1. Knowledge (about second scenario): single categorical item assessing understanding of the information: 'As people get older, they may no longer benefit from screening tests' ('True', 'False' and 'Don't know').

\section{Demographics and health information}

Gender, age, relationship status, education, general health, screening history, perceived risk and family history of breast/prostate cancer, activities of daily living, ${ }^{26}$ competing morbidities ${ }^{36}$ and health literacy ${ }^{37}$ were measured.

\section{Sample size}

The recruitment target was 252 participants aged 65 years and older, which was sufficient based on a sample size calculation using Cohen's $\mathrm{f}$ formula (ie, the ratio of the SD of group means and the SD within groups) informed by preliminary data of the primary outcome (screening intention) to detect a moderate effect size $(\mathrm{f}=0.25)$ with $80 \%$ power and $\alpha$ at 0.025 . As such, the minimum target for each of the 4 groups was 64 participants. Groups of this size would also provide $80 \%$ power to detect pairwise differences between groups of 0.55 SDs (corresponding to $\sim 1.4$ units on a 10-point scale) at $\alpha$ of 0.025 for the primary outcome of screening intention.

\section{Statistical methods}

Analysis was conducted using SPSS V.22.0. Before analysis, data were checked for missing or outlying values. Since the information about PSA testing and mammography screening was subtly different (eg, test procedure, treatment), we checked for gender differences in primary outcomes. On average, men reported significantly higher screening intention than women, but there was no difference for cancer anxiety. Therefore, screening intention was analysed using analysis of covariance (ANCOVA), controlling for gender, and cancer anxiety was analysed using analysis of variance. Furthermore, considering the new and exploratory nature of the study, post-hoc contrasts were used to identify unexpected effects, with an $\alpha$ level of 0.05 used for all tests.
RESULTS

\section{Participants}

Recruitment took place from 7 to 17 August 2018. The number of participants who were randomised, received the intervention and were analysed for primary outcomes is shown in figure 1 . The trial ended once the target sample size was reached and final analysis included 271 responses. The age range was $65-90$ years $(\mathrm{M}=71.9$, $\mathrm{SD}=5.6)$, over two-thirds were married $(69.7 \%)$ and half had completed a university degree, diploma or certificate $(50.6 \%)$. Many participants reported good or very good general health $(68.6 \%)$, most did not have problems with activities of daily living $(77.9 \%)$ and level of health literacy was heterogeneous across the sample, including 78 participants $(28.8 \%)$ with low level (meaning they made more than one error when interpreting a fictitious medical label). ${ }^{37}$ Half of the sample had engaged in mammography or PSA screening in the last 12 months $(50.9 \%)$ and most participants perceived that they had a low chance of developing breast or prostate cancer in their lifetime $(69.0 \%)$. Just under a quarter reported one or more family members (of the same gender) had been diagnosed with breast or prostate cancer $(24.0 \%)$. Demographic and health information by condition are reported in table 1 . There were no differences across groups.

\section{Screening intention}

Overall, there was no significant main effect of condition on screening intention. However, a post-hoc contrast of the greatest difference (figure 2) found that screening intention was significantly lower for those who read the negatively framed life expectancy statement than those who read the health status statement at time 1 ( $6.0 \mathrm{vs} 7.1$, adjusted mean difference $(\mathrm{MD})=1.1, \mathrm{p}=0.049,95 \%$ CI 0.0 to 2.2).

\section{Cancer anxiety}

The main effect of condition on cancer anxiety was not significant. However, post-hoc contrasts testing the greatest difference (figure 2) found that cancer anxiety was significantly higher for those who read the positively framed life expectancy statement compared with those who read the negatively framed life expectancy statement ( 4.8 vs $5.8, \mathrm{MD}=1.0, \mathrm{p}=0.025,95 \% \mathrm{CI} 0.1$ to 1.9 ).

\section{Effect of anchoring bias intervention on screening intention and cancer anxiety}

The within-subjects effects were significant, such that screening intention significantly decreased (figure 3) after the anchoring bias intervention when collapsed across conditions (adjusted $\mathrm{MD}=0.8, \mathrm{~F}(1,266)=4.11$, $\mathrm{p}=0.044$, CI 0.6 to 1.0$)$. The same pattern was observed for cancer anxiety $(\mathrm{MD}=0.3, \mathrm{~F}(1,267)=9.50, \mathrm{p}=0.002$, CI 0.1 to 0.4$)$.

\section{Secondary outcomes}

Trust

There was no significant main effect of condition on trust in the information provided, trust in GP or trust in the 
Table 1 Demographic characteristics and health information by condition $(n=271, n(\%)$ unless otherwise stated)

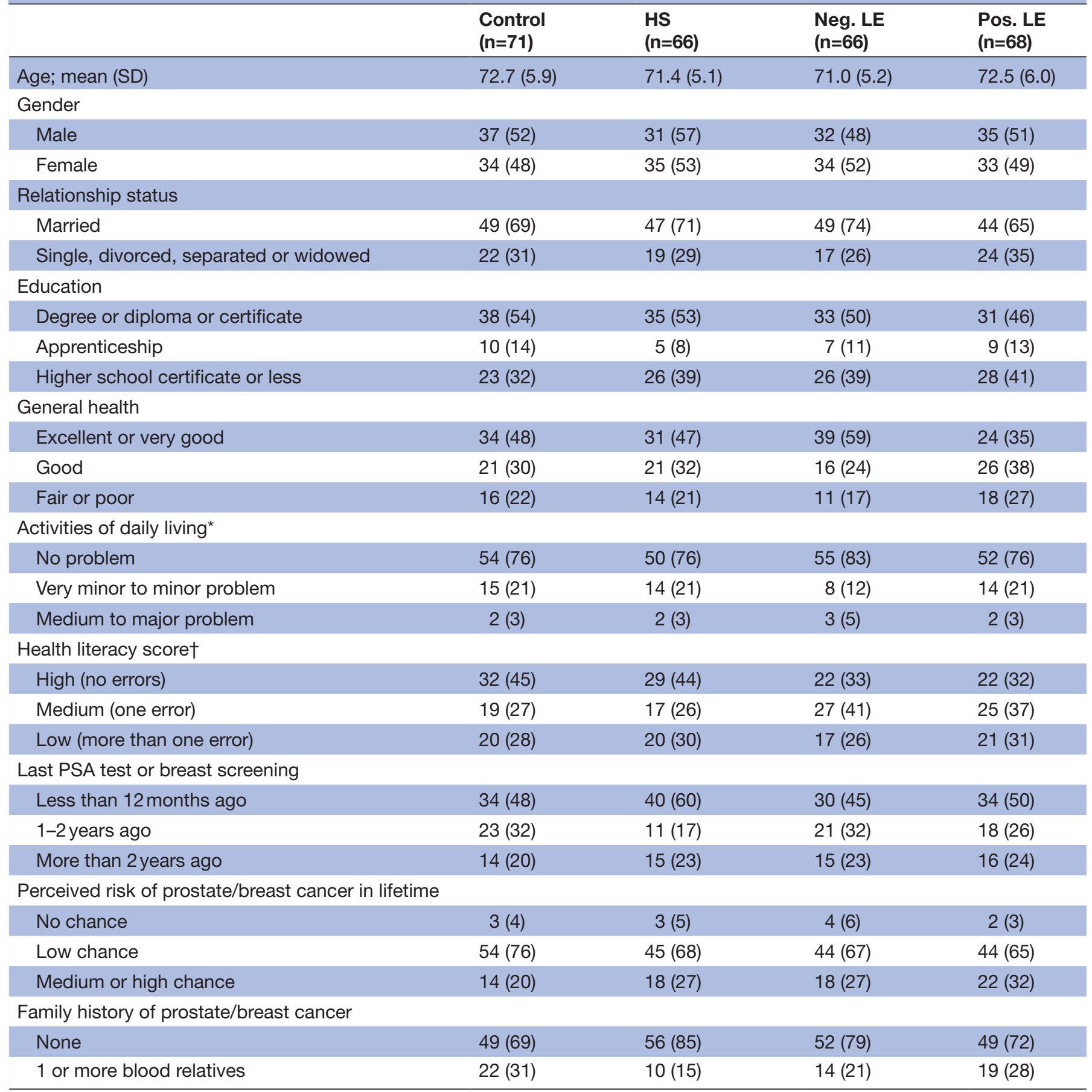

*Activities of daily living: no problem=no daily tasks found difficult (0/5), very minor to minor=some daily tasks found difficult (1-2/5), medium to major=most daily tasks found difficult $(3-5 / 5)$.

†Health literacy: high=scored $4 / 4$, medium=3/4, low=scored $2 / 4,1 / 4$ or $0 / 4$ when asked to interpret a medical label.

HS, health status condition; Neg. LE, negatively framed life expectancy condition; Pos. LE, positively framed life expectancy condition.

healthcare system. However, post-hoc contrasts found that trust in GP was significantly lower for those who read the positively framed life expectancy statement compared with those who read the negatively framed life expectancy statement $(\mathrm{MD}=0.3, \mathrm{p}=0.037,95 \% \mathrm{CI} 0.0$ to 0.5$)$ or control statement (MD=0.3, $\mathrm{p}=0.022,95 \% \mathrm{CI} 0.0$ to 0.5 ; figure 4$)$. Post-hoc contrasts for trust in the information provided and trust in the healthcare system were not significant.
Average trust in the information provided was also high overall $($ mean $=4.2$, range $1-5)$.

Decisional conflict

There was a significant main effect of condition on the values clarity subscale of the decisional conflict scale $(\mathrm{F}(3$, $267)=2.83, p=0.039$ ), suggesting clarity of values relating to the screening decision differed depending on the 


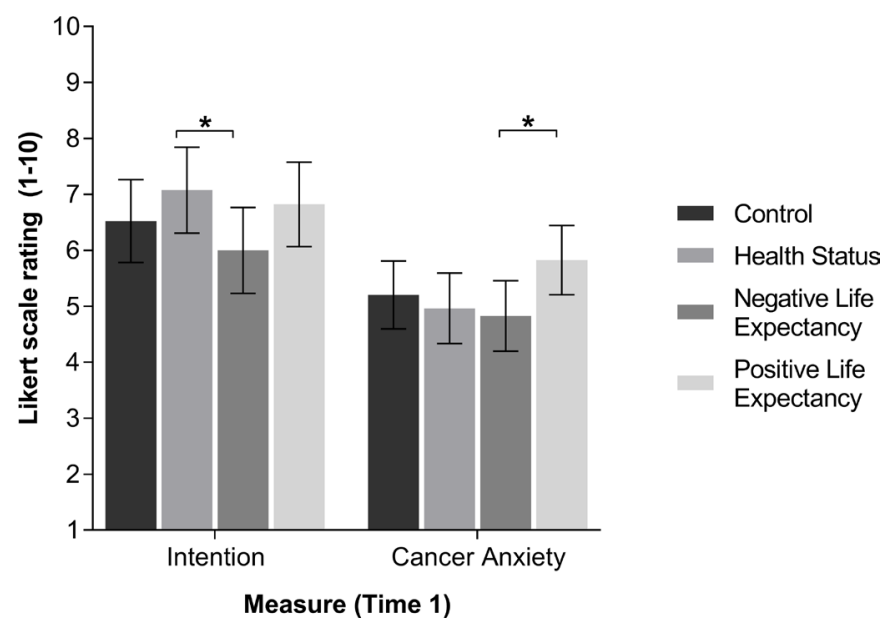

Figure 2 Adjusted mean intention and unadjusted mean cancer anxiety $( \pm 95 \%$ Cls) by condition at time $1(n=271$, range $=1-10)$. ${ }^{\star}$ Contrast $p<0.05$.

recommendation statement received (figure 5). Post-hoc contrasts found those who read the positively framed life expectancy statement were less clear about their values related to their screening decision than those who read the negatively framed life expectancy $(\mathrm{MD}=8.2, \mathrm{p}=0.027$, $95 \%$ CI 0.1 to 15.5$)$ or health status statement $(\mathrm{MD}=7.9$, $\mathrm{p}=0.034,95 \%$ CI 0.6 to 15.1 ). Those who read the negatively framed life expectancy statement felt significantly clearer about their values than those who read the control statement $(\mathrm{MD}=7.2, \mathrm{p}=0.049,95 \%$ CI 0.0 to 14.4$)$. There were no significant main effects or post-hoc contrasts for total decisional conflict or the other subscales (uncertainty, informed and support).

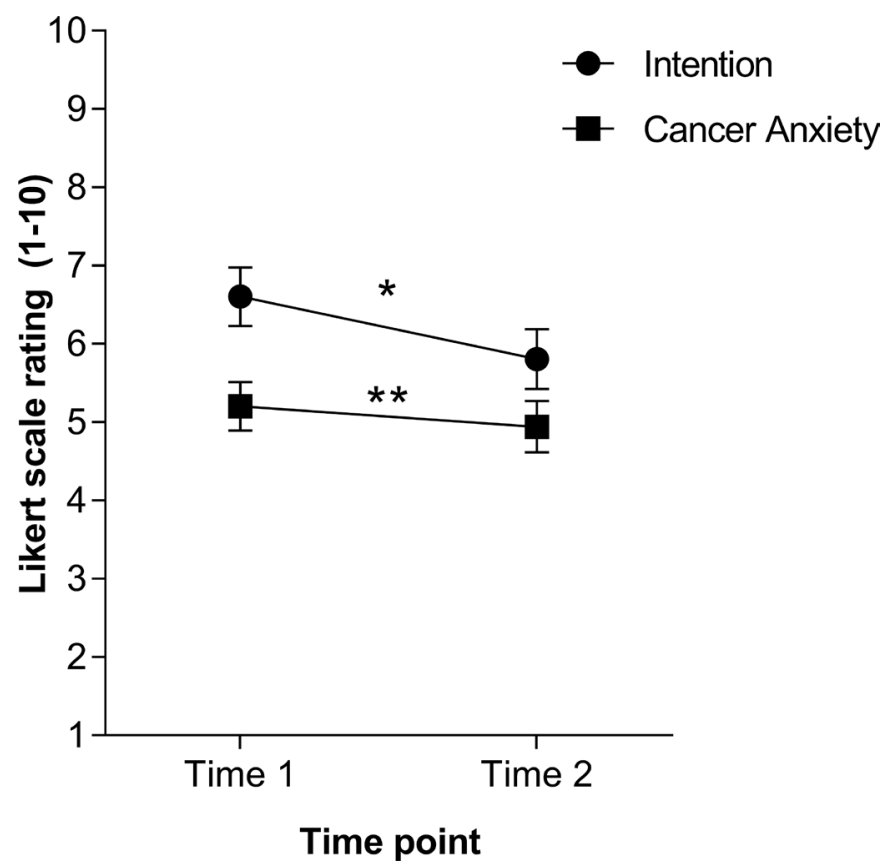

Figure 3 Means for primary outcomes ( $\pm 95 \% \mathrm{Cls}$ ) pre (time 1) and post (time 2) anchoring bias intervention ( $n=271$, range $=1-10) .{ }^{* \star} \mathrm{P}<0.01 .{ }^{*} \mathrm{P}<0.05$.

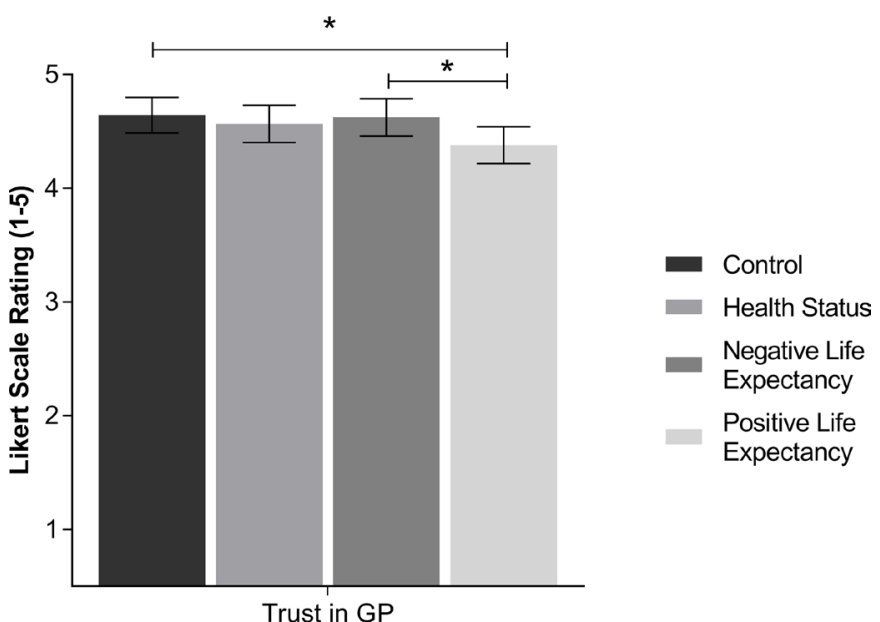

Figure 4 Mean trust in general practitioner (GP) scores ( $\pm 95 \%$ Cls) by condition at time $1(n=271$, range $1-7)$; ${ }^{*}$ Contrast $\mathrm{p}<0.05$.

Knowledge

A $\chi^{2}$ test indicated that knowledge about the mortality benefit of screening (first scenario) did not differ significantly depending on recommendation statement at time $1\left(\chi^{2}=2.30, p=0.514\right)$, nor did knowledge about screening in older adults (second scenario) at time $2\left(\chi^{2}=6.35\right.$, $\mathrm{p}=0.096)$. Just over two-thirds $(67.2 \%)$ of participants correctly answered the knowledge question at time 1 (mortality benefits), and only half $(48.0 \%)$ of participants correctly answered the knowledge question at time 2 (screening in older adults).

\section{DISCUSSION}

In our study, older adults reported the lowest screening intention when they received the scenario in which the GP communicated that they may not live long enough to benefit from cancer screening. Screening intention in this group was significantly lower than the group who were told that their other health issues should take priority. This group also had lower cancer anxiety, higher trust in

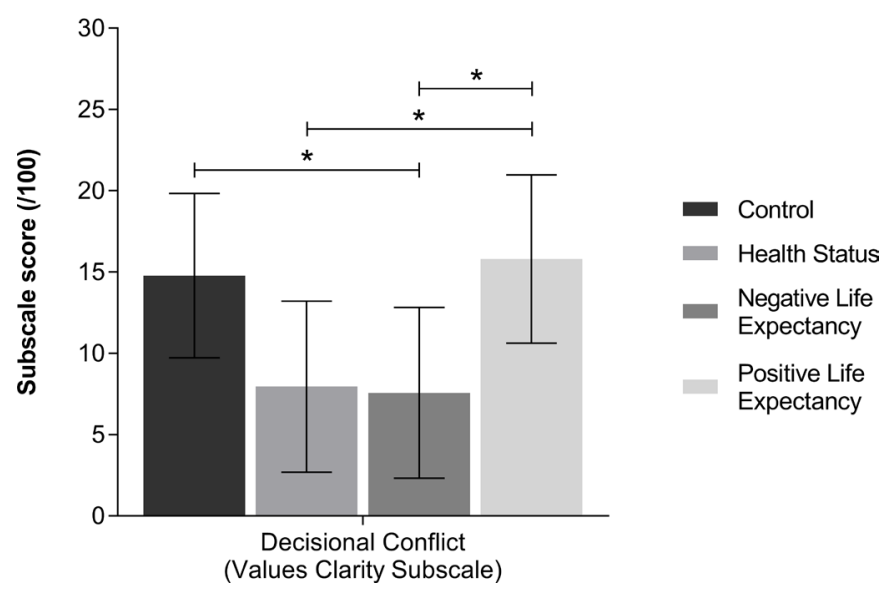

Figure 5 Mean decisional conflict values clarity subscale scores $( \pm 95 \%$ Cls) by condition at time $1(n=271$, range $0-100)$. *Contrast $\mathrm{p}<0.05$. 
the GP and felt clearer about their values related to the screening decision, compared with the group who were told that the test would not help them live longer. After the GP explained how and why this information contradicts previous positive messages about the benefits of cancer screening (addressing anchoring bias) ${ }^{29}$ both screening intention and cancer anxiety decreased in all groups.

Research has shown that some older adults consider their life expectancy irrelevant when discussing stopping cancer screening because of their scepticism around life expectancy predictions and the worry such information could cause, ${ }^{26}$ and in some circumstances this information may be perceived as discriminatory. ${ }^{38}$ However, we found that participants who were told they may not live long enough to benefit from screening reported the lowest screening intention without adverse effects in terms of increased cancer anxiety or reduced trust or reduced clarity of values related to the screening decision. In contrast, those who were told the test would not help them live longer reported the highest cancer anxiety, lowest trust in GP and lower clarity of values, demonstrating the importance of message framing, as suggested in previous qualitative work comparing older adults' and clinicians' perspectives. ${ }^{25}$ Additionally, previous qualitative findings regarding the impact of recommendations to stop screening on trust have been mixed. In one study, participants reported that if they would receive a recommendation to stop screening, it would likely threaten their trust in their clinician. ${ }^{39}$ On the other hand, more recent work examining older adults' perspectives suggested they may not have negative reactions to such a recommendation, particularly in the context of an existing trusting relationship with their clinician. ${ }^{25}$ However, there was still great concern for negative reactions reported from the clinician perspective.$^{25}$ Importantly, our quantitative findings are the first to suggest that certain framings of recommendations to stop screening may differentially impact trust in the patient-physician relationship (although conclusions are limited by the fact that trust was not measured at baseline).

Although the 'harsher' life expectancy statement was least preferred by older adults in previous research, ${ }^{27}$ this strategy should not be discounted by clinicians deciding how to communicate a recommendation to stop screening. The confronting nature could be needed to help override older adults' strong biases in favour of screening and enable the option of stopping screening to be considered, while also avoiding any adverse psychological effects of increased anxiety. This finding is important, as educating older adults about the potential harms of screening may not be sufficient to change their screening behaviours. ${ }^{40}$ Despite clinicians being hesitant to incorporate life expectancy into discussions about stopping screening, some older adults think that a discussion incorporating life expectancy is necessary. ${ }^{25}$ Perhaps a clinician could use this confronting statement in the context of a trusting relationship where the life expectancy of an older patient is clearly limited, but they have a strong desire to continue screening. However, further studies are needed to test clinicians' acceptability of these communication strategies. Future studies should also examine the effects of a confronting communication strategy in a clinical older adult sample, as our online panel sample may have been more amenable to such an approach because they may be more engaged with medical research and knowledge. Alternatively, the more neutral control statement communicating the test would harm you more than benefit you alone might also be a good strategy given that our study found insufficient evidence to suggest it differed from the harsher statement. However, this needs to be examined in a future study with a bigger sample size.

Our findings also suggest that if clinicians communicate to older adults about stopping screening in the way they prefer (ie, incorporating health status), ${ }^{26}$ this may not be sufficient to override their strong belief in the benefits of continuing screening and change their screening behaviour. This could be due to some older adults' belief that cancer screening is always beneficial, or even needed more, for those with poor health. ${ }^{26}$ This discrepancy between preferences and what best supports decisionmaking has also been shown in other risk communication contexts. ${ }^{41} 42$

Importantly, the consistent differences in outcomes between the negatively framed life expectancy statement and the other statements could also be explained by the uncertainty conveyed (you may not live long enough to benefit). All other statements instead conveyed a degree of certainty, which may have triggered a negative reaction, especially in a highly uncertain context such as screening. Older adults are likely sceptical towards their clinicians' estimation of how long they will live, ${ }^{26}$ so communicating certainty regarding life expectancy specifically could have had a negative impact. However, older adults report greater preferences for these more certain statements, ${ }^{26}$ which warrants further investigation of the effects of the certainty of these recommendation statements.

A recommendation to stop screening could also be communicated sensitively if clinicians take the time to explicitly acknowledge why it contradicts previous positive screening messages, as screening intention and cancer anxiety were significantly lower for all participants following the anchoring bias intervention. This finding is compatible with previous work that suggests forewarning a patient of their anchoring bias when communicating disease risk can reduce its effect. ${ }^{43}$ Similarly, explicitly explaining how and why the screening recommendation has changed, in line with crisis communication guidelines ${ }^{29}$ may be a useful strategy for reducing the anchoring bias in this context. However, future work should examine the effect of this strategy alone, as it is not clear that this intervention would be more effective than another type of intervention that reinforces the information provided beforehand.

Overall, our findings indicate that supporting older adults to make an informed decision about cancer screening, where the options of continuing or stopping 
are both considered, is challenging. Participants reported high overall cancer screening intentions. Interestingly, men reported higher intentions than women overall, which is surprising considering the controversy surrounding PSA testing. ${ }^{44}$ Participants also demonstrated limited knowledge about the potential harms of continuing screening. This is not surprising, as older people have long heard persuasive, positive messages about the benefits of screening, and have great faith in the importance of adhering to preventive healthcare as a way of looking after themselves. ${ }^{45}$ Consequently, the idea of not needing screening anymore may be difficult to consider ${ }^{39}$ hindering the uptake of new information about the potential harms of continuing. ${ }^{246}$ Despite these challenges, our findings suggest that a more confronting, less preferred communication strategy may be more effective than more preferred strategies to enable older adults to consider the option of stopping screening.

\section{Limitations}

Our study has several limitations. First, it may have been underpowered to detect statistically significant differences. Our a priori sample size calculation was based on screening intention reported in a substantially younger sample, as no similar quantitative work has previously been done in an older population. Older people have more varied health than younger people and may report more varied screening intention, so more participants may have been required. Given the exploratory nature of our study and the lack of context to interpret our findings, it is difficult to make clear judgements of their clinical importance. However, we suggest that a one-point difference on a 10-point scale for both primary outcomes is clinically important. This interpretation regarding the negatively framed life expectancy statement, for example, is further supported by its' consistently favourable differences across all five outcomes, as well as the fact one might have initially expected to find adverse effects. Moreover, the effect sizes from this study can now inform the planning of future studies where stronger conclusions about any differences in the effect of the statements can be made. We also acknowledge the limitation of our analysis given the issue of multiple testing and suggest that future studies use planned comparisons in a larger sample to draw firmer conclusions in an otherwise novel area of research.

Our study is also limited because we measured intention rather than actual behaviour and presented hypothetical scenarios. Although these scenarios would not realistically replicate the experience of an actual conversation with a GP, we designed their content with the advice of clinician experts to ensure a conversation was accurately depicted. Individuals in a real-life setting may behave differently. Despite this limitation, the data we collected (including free-text comments at the end of the survey) demonstrated that participants were highly engaged with the content and felt high trust in the information provided. Our study was also limited as we did not measure baseline outcomes before the recommendation, which future studies should consider. Finally, despite Dynata's large panel being increasingly used in recruiting for online research, the generalisability of our older adult sample may have been limited due to this recruitment method. Our sample was quite diverse in terms of sociodemographic characteristics, but future work in a larger, more diverse community-based sample is warranted. For example, tailoring statements to different subsets of older adults (based on factors such as health status, life expectancy and age) might better enable informed screening decisions in this population.

\section{CONCLUSIONS}

Our findings suggest that older adults may be more open to consider stopping screening without experiencing greater cancer anxiety when clinicians communicate that they may not live long enough to benefit from screening and explicitly explain how and why this information contradicts previous positive messages about cancer screening. Despite the challenges of communicating about stopping, this option ought to be communicated, especially to older people who could be more likely to experience harm if they continue screening. For further insight into this sensitive and challenging area of research, future studies should both quantitatively and qualitatively explore what drives older adults' cancer screening decision-making and examine how different communication strategies in this setting influence informed choice in larger samples. This may help to reduce the negative impact of overdiagnosis and unnecessary treatment in the older population.

Twitter Jenna Smith @smith_jenna_, Jolyn Hersch @jolynhersch and Erin Cvejic @ ErinCvejic

Acknowledgements The authors would like to thank Paul Glasziou for helpful input regarding the scenarios, Julie Ayre for support using Qualtrics, Dynata for recruitment services and all study participants.

Contributors JS, JJ, RHD, JH and KM conceived the study, were involved in the design of the study, the methods development and drafting of the manuscript. JS coordinated the running of the study. JS and EC contributed to the data analysis. JS drafted the manuscript. All authors contributed to the interpretation of the analysis and critically revised the manuscript.

Funding This work was supported by NHMRC Program Grant (APP1113532) and NHMRC CDF Fellowship (APP1162149).

\section{Competing interests None declared.}

Patient and public involvement Patients and/or the public were not involved in the design, or conduct, or reporting, or dissemination plans of this research.

\section{Patient consent for publication Not required.}

Ethics approval The study was approved by the University of Sydney Human Research Ethics Committee (2018/357).

Provenance and peer review Not commissioned; externally peer reviewed.

Data availability statement Data are available upon reasonable request. Deidentified participant data will be made available upon request to anyone wishing to access it who provides a methodologically sound proposal to the principal investigator. Contact details of the principal investigator are: jesse.jansen@ maastrichtuniversity.nl

Open access This is an open access article distributed in accordance with the Creative Commons Attribution Non Commercial (CC BY-NC 4.0) license, which permits others to distribute, remix, adapt, build upon this work non-commercially, 
and license their derivative works on different terms, provided the original work is properly cited, appropriate credit is given, any changes made indicated, and the use is non-commercial. See: http://creativecommons.org/licenses/by-nc/4.0/.

\section{ORCID iDs}

Jenna Smith http://orcid.org/0000-0002-0480-8125

Rachael H Dodd http://orcid.org/0000-0002-8080-6359

Jolyn Hersch http://orcid.org/0000-0001-5225-6639

Erin Cvejic http://orcid.org/0000-0002-6043-6071

\section{REFERENCES}

1 Breslau ES, Gorin SS, Edwards HM, et al. An individualized approach to cancer screening decisions in older adults: a multilevel framework. J Gen Intern Med 2016;31:539-47.

2 Clarfield AM. Screening in frail older people: an ounce of prevention or a pound of trouble? J Am Geriatr Soc 2010;58:2016-21.

3 Walter LC, Covinsky KE. Cancer screening in elderly patients: a framework for individualized decision making. JAMA 2001;285:2750-6.

4 Moyer VA. Screening for prostate cancer: U.S. preventive services Task force recommendation statement. Ann Intern Med 2012;157:120-34.

5 Ilic D, Neuberger MM, Djulbegovic M, et al. Screening for prostate cancer. Cochrane Database Syst Rev 2013;107.

6 Martin RM, Donovan JL, Turner EL, et al. Effect of a low-intensity PSA-based screening intervention on prostate cancer mortality: the cap randomized clinical trial. JAMA 2018;319:883-95.

7 Jacklyn G, Howard K, Irwig L, et al. Impact of extending screening mammography to older women: information to support informed choices. Int J Cancer 2017;141:1540-50.

8 Gunsoy NB, Garcia-Closas M, Moss SM. Estimating breast cancer mortality reduction and overdiagnosis due to screening for different strategies in the United Kingdom. Br J Cancer 2014;110:2412.

9 Walter LC, Schonberg MA. Screening mammography in older women: a review. JAMA 2014;311:1336-47.

10 Welch HG, Black WC. Overdiagnosis in cancer. J Natl Cancer Inst 2010;102:605-13.

11 Sanda MG, Dunn RL, Michalski J, et al. Quality of life and satisfaction with outcome among prostate-cancer survivors. $N$ Engl J Med 2008;358:1250-61.

12 Moynihan R, Doust J, Henry D. Preventing overdiagnosis: how to stop harming the healthy. BMJ 2012;344:e3502.

13 Oeffinger KC, Fontham ETH, Etzioni R, et al. Breast cancer screening for women at average risk: 2015 guideline update from the American cancer Society. JAMA 2015;314:1599-614.

14 Tonelli M, Connor Gorber S, Joffres M, et al. Recommendations on screening for breast cancer in average-risk women aged 40-74 years. CMAJ 2011;183:1991-2001.

15 BreastScreen Australia. Breastscreen Australia national accreditation standards, 2015.

16 Prostate Cancer Foundation of Australia and CancerCouncil Australia. Clinical practice guidelines for PSA testing and early management of test-detected prostate cancer. Prostate Cancer Foundation of Australia and Cancer Council Australia PSA Testing Guidelines Expert Advisory Panel, 2016.

17 National Health Service (NHS) Choice UK. Prostate cancer - PSA screening, 2015

18 Royce TJ, Hendrix LH, Stokes WA, et al. Cancer screening rates in individuals with different life expectancies. JAMA Intern Med 2014;174:1558-65

19 Kotwal AA, Walter LC, Lee SJ, et al. Are we choosing wisely? older adults' cancer screening intentions and recalled discussions with physicians about stopping. J Gen Intern Med 2019;34:1538-1545.

20 Woloshin S, Schwartz LM, Black WC, et al. Cancer screening campaigns--getting past uninformative persuasion. N Engl J Med 2012;367:1677-9.

21 Entwistle VA, Carter SM, Trevena L, et al. Communicating about screening. BMJ 2008;337:a1591.

22 Schoenborn NL, Xue Q-L, Pollack CE, et al. Demographic, health, and attitudinal factors predictive of cancer screening decisions in older adults. Prev Med Rep 2019;13:244-8.
23 McCaffery KJ, Jansen J, Scherer LD, et al. Walking the tightrope: communicating overdiagnosis in modern healthcare. BMJ 2016;352:i348.

24 Schoenborn NL, Bowman TL, Cayea D, et al. Discussion strategies that primary care clinicians use when stopping cancer screening in older adults. J Am Geriatr Soc 2016;64:e221-3.

25 Schoenborn NL, Boyd CM, Lee SJ, et al. Communicating about stopping cancer screening: comparing clinicians' and older adults' perspectives. Gerontologist 2019;59:S67-76.

26 Schoenborn NL, Lee K, Pollack CE, et al. Older adults' views and communication preferences about cancer screening cessation. JAMA Intern Med 2017;177:1121-8.

27 Schoenborn NL, Janssen EM, Boyd CM, et al. Preferred clinician communication about stopping cancer screening among older us adults: results from a national survey. JAMA Oncol 2018;4:1126-8.

28 Hersch J, Barratt A, Jansen J, et al. Use of a decision aid including information on overdetection to support informed choice about breast cancer screening: a randomised controlled trial. Lancet 2015;385:1642-52.

29 Sandman P, Lanard J. Crisis communication: guidelines for action, 2004: 20.

30 Copp T, McCaffery K, Azizi L, et al. Influence of the disease label 'polycystic ovary syndrome' on intention to have an ultrasound and psychosocial outcomes: a randomised online study in young women. Hum Reprod 2017;32:876-84.

31 Scherer LD, Shaffer VA, Caverly T, et al. The role of the affect heuristic and cancer anxiety in responding to negative information about medical tests. Psychol Health 2018;33:292-312.

32 Hillen MA, de Haes HCJM, Verdam MGE, et al. Does source of patient recruitment affect the impact of communication on trust? Patient Educ Couns 2014:95:226-30.

33 O'Connor AM. Validation of a decisional conflict scale. Med Decis Making 1995;15:25-30.

34 Fraenkel L, Street RL, Towle V, et al. A pilot randomized controlled trial of a decision support tool to improve the quality of communication and decision-making in individuals with atrial fibrillation. J Am Geriatr Soc 2012;60:1434-41.

35 Eden KB, Scariati P, Klein K, et al. Mammography decision aid reduces decisional conflict for women in their forties considering screening. J Womens Health 2015;24:1013-20.

36 Katz JN, Chang LC, Sangha O, et al. Can comorbidity be measured by questionnaire rather than medical record review? Med Care 1996;34:73-84.

37 Bostock S, Steptoe A. Association between low functional health literacy and mortality in older adults: longitudinal cohort study. BMJ 2012;344:e1602

38 Degeling C, Barratt A, Aranda S, et al. Should women aged 70-74 be invited to participate in screening mammography? A report on two Australian community juries. BMJ Open 2018;8:e021174.

39 Torke AM, Schwartz PH, Holtz LR, et al. Older adults and forgoing cancer screening: "I think it would be strange". JAMA Intern Med 2013;173:526-31.

40 Gross CP, Fried TR, Tinetti ME, et al. Decision-Making and cancer screening: a qualitative study of older adults with multiple chronic conditions. J Geriatr Oncol 2015;6:93-100.

41 McCaffery KJ, Dixon A, Hayen A, et al. The influence of graphic display format on the interpretations of quantitative risk information among adults with lower education and literacy: a randomized experimental study. Med Decis Making 2012;32:532-44.

42 Feldman-Stewart D, Kocovski N, McConnell BA, et al. Perception of quantitative information for treatment decisions. Med Decis Making 2000;20:228-38.

43 Senay I, Kaphingst KA. Anchoring-and-adjustment bias in communication of disease risk. Med Decis Making 2009;29:193-201.

44 Pickles K, Carter SM, Rychetnik L. Doctors' approaches to PSA testing and overdiagnosis in primary healthcare: a qualitative study. BMJ Open 2015;5:e006367.

45 Jansen J, McKinn S, Bonner C, et al. Shared decision-making about cardiovascular disease medication in older people: a qualitative study of patient experiences in general practice. BMJ Open 2019;9:e026342.

46 Scherer LD, Valentine KD, Patel N, et al. A bias for action in cancer screening? J Exp Psychol Appl 2019;25:149-61. 